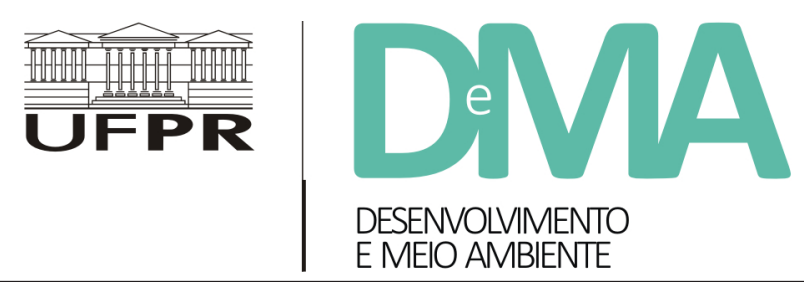

\title{
Modelos propositivos para gestão pesqueira e ambiental na região do Albardão, sul do Rio Grande do Sul
}

\section{The Propositional Models for Fisheries and Environmental Management in the Albardão Region, South of Rio Grande do Sul State}

\author{
Paulo Henrique MATTOS $^{1 *}$, Washington Luiz dos Santos FERREIRA ${ }^{2}$ \\ ${ }^{1}$ Universidade Federal do Rio Grande (FURG), Rio Grande, RS, Brasil. \\ ${ }^{2}$ Universidade Federal de Santa Catarina (UFSC), Florianópolis, SC, Brasil. \\ *E-mail de contato: mattos.paulo@gmail.com
}

Artigo recebido em 31 de agosto de 2017, versão final aceita em 18 de dezembro de 2017.

\begin{abstract}
RESUMO: A região do "Albardão", no litoral do extremo sul do Brasil, é reconhecida por sua expressiva produtividade pesqueira, resultante da dinâmica dos processos oceanográficos regionais, que garantiu o desenvolvimento de uma diversificada frota pesqueira, que abastece os parques industriais de Rio Grande (RS) e Itajaí (SC) e sustenta parte da frota pesqueira de Santos (SP), além de grande contingente de embarcações do exterior. Esta região também sustenta grande biodiversidade e muitas espécies de peixes, aves, mamíferos e tartarugas marinhas ameaçadas de extinção, pela captura acidental ou direcionada por meio da intensa atividade pesqueira. Dentre este conjunto de conflitos socioambientais, destaca-se a recente proposição de criação de uma Unidade de Conservação (UC) marinha na região. Caso a implantação desta UC ocorra conforme os planos oficiais, prevê-se o incremento nos conflitos, em função da restrição de acesso das comunidades de pesca artesanal às áreas e aos recursos pesqueiros e à supressão da atividade pela frota industrial em grandes áreas marinhas adjacentes. Esta opção provocaria contundente rejeição social e política aos esforços conservacionistas, dificultando a efetiva implantação da UC proposta, especialmente devido aos casos similares, implantados sem considerar os direitos de acesso dos usuários às suas áreas de histórica atuação. De modo a subsidiar este processo quanto às suas melhores opções socioambientais, foi organizada intensiva revisão bibliográfica e análise documental das publicações disponíveis sobre o tema e a região; procedeu-se à elaboração de um Sistema de Informações Geográficas e à estruturação dos cenários conceituais preditivos sobre os potenciais desdobramentos da proposta (sem a implantação da UC; com a implantação da UC como Parque Nacional; com a implantação como um Mosaico de UCs). Ao final, procedeu-se à argumentação do custo-benefício da opção entendida como a mais coerente (o Mosaico de UCs), desdobrando-a em dois subconjuntos, de menor (a) e maior (b) abrangência e relevância socioambiental.
\end{abstract}


Palavras-chave: gestão pesqueira e ambiental; Albardão; modelos propositivos.

ABSTRACT: The "Albardão" region, located on the extreme south coast of Brazil, is recognized for its expressive fishing productivity, resulting from the dynamics of the regional oceanic processes, which guarantees the development of a diversified fishery, one that supplies the industrial parks of Rio Grande (RS) and Itajaí (SC) and supports part of the fishing fleet of Santos (SP), besides a large contingent of foreign vessels. This region also has a great biodiversity and many endangered species of fish, birds, marine mammals and sea turtles, through bycatch or directed capture from intense fishing activity. Among this set of socioenvironmental conflicts, a recent proposal for the creation of a Marine Conservation Unit (UC) in the region stands out. If the implementation of the UC takes place according to the official plans, an increase in conflicts is predicted due to the restriction of access to fishing areas and resources for artisanal fishing communities and the suppression of activity by industrial fleet in large adjacent marine areas. This option would provoke a strong social and political rejection of conservation efforts, making it difficult to effectively implement UC's proposal, especially due to similar cases without considering user rights to access their areas. In order to subsidize this process regarding the best socioenvironmental options, an intensive bibliographical review and documentary analysis of the available publications on the theme and region was organized; a Geographic Information System was elaborated and the conceptual predictive scenarios were structured on the potential unfolding of the proposal (without the implementation of the UC; with the implementation as a National Park; and with the implementation as a Mosaic of UCs). The cost-benefit argument was developed for the most coherent option chosen (the Mosaic of UCs), unfolding it into two subsets, of smaller (a) and greater (b) scope and socioenvironmental relevance.

Keywords: fisheries and environmental management; Albardão Region; propositional models.

\section{Introdução}

A plataforma continental do Rio Grande do Sul (RS) configura-se em um sítio geográfico de elevadas biodiversidade e produtividade biológica, devido ao contínuo aporte de nutrientes das bacias hidrográficas do Sistema Lagunar Patos-Mirim (Brasil) e do Rio da Prata (Uruguai e Argentina), assim como da alternância sazonal das correntes do Brasil e das Malvinas (Niencheski \& Windom, 1994; Garcia, 1998; Attisano et al., 2008; Piola et al., 2000; Silva Jr. et al., 1996; Vaz et al., 2006). Esta produtividade biológica repercute diretamente na acentuada produção pesqueira (Chao et al., 1985; Haimovici et al., 1996; Schroeder \& Castello, 2007). Tal significado biogeográfico, ecológico e econômico é particularmente relevante na sua porção meridional, conhecida como Albardão (Figura 1).

Esta relevância é decorrente dos fenômenos de transporte subterrâneo de férteis águas lagunares (Attisano et al., 2008) que atingem a costa, assim como pela heterogeneidade das feições e pela topografia subaquosa, que promovem o aumento da produção primária costeira, sustentando elevadas abundância e diversidade de recursos pesqueiros. Essa costa é formada por praias arenosas expostas e sem reentrâncias, com declividade suave, contendo principalmente sedimentos terrígenos.

Tais áreas foram indicadas como extremamente importantes para a conservação, segundo o projeto "Avaliação e Ações Prioritárias para a Conservação da Biodiversidade das Zonas Costeira e Marinha", elaborado em 2007 pelo MMA, por serem áreas de transição de espécies tropicais e 


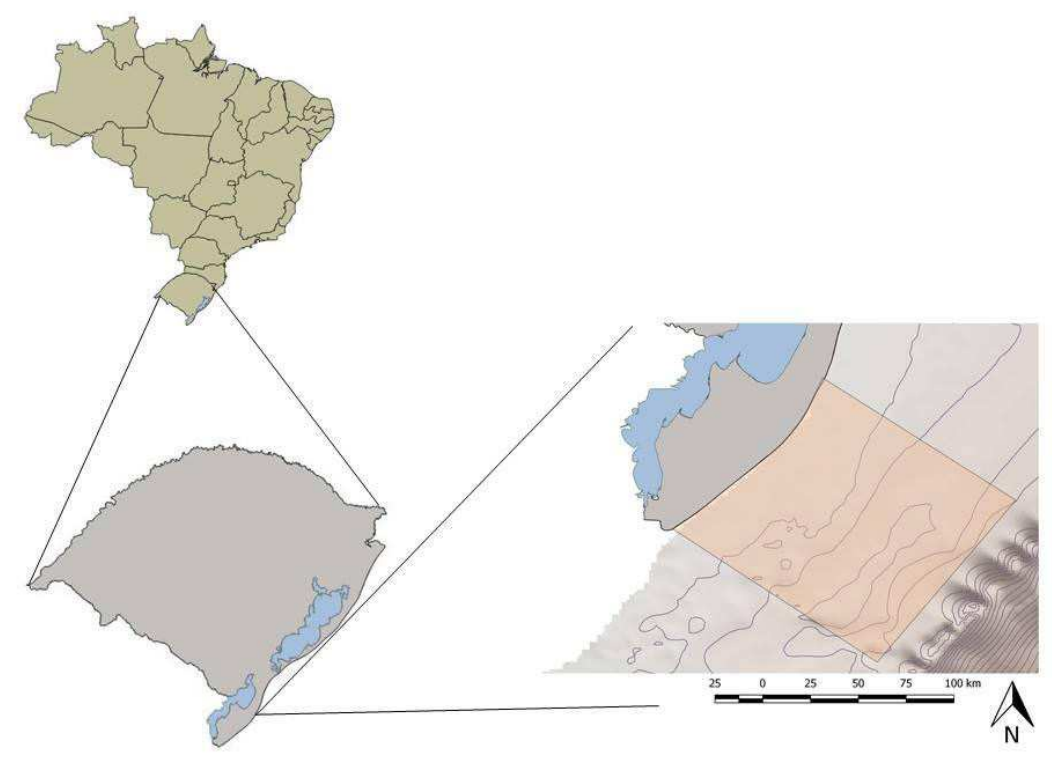

FIGURA 1 - Região do Albardão, litoral do extremo sul do Brasil.

temperadas, bem como áreas de concentração de pequenos cetáceos, como a toninha (Pontoporia blainvillei), berçário e agregação de diversas espécies de elasmobrânquios, dentre elas cerca de 20 espécies ameaçadas (Vooren \& Klippel, 2005a), área de ocorrência de diversas espécies de albatrozes e petréis, especialmente a pardela-de-óculos (Procellaria conspicillata), criticamente ameaçada, e de juvenis do albatroz-de-sobrancelha-negra (Thalassarche melanophris) (Bugoni et al., 2009).

Além disso, estes ambientes estão sujeitos à alta pressão antrópica, em virtude do grande desenvolvimento da pesca artesanal e industrial, fazendo com que os estoques regionais apresentem sinais de sobre-explotação, como a depleção dos elasmobrânquios e espécies ameaçadas de tartarugas, aves e mamíferos marinhos, capturadas acidentalmente pela pesca (Secchi et al., 1997; Bugoni et al., 2008; Mancini et al., 2009; Ferreira et al., 2010).

A região do Albardão já foi objeto de propostas anteriores, como a proposta de criação de Áreas de Exclusão de Pesca na região costeira por ser, esta, berçário de espécies ameaçadas pela sobrepesca, como Squatina oculta, S. guggenheim e Mustelus fasciatus. Para a conservação dessas espécies, segundo Vooren \& Klippel (2005b), a melhor alternativa é que as pescarias com captura incidental sejam excluídas das áreas críticas dessas espécies, ou de uma parte dessas áreas.

Outra proposta mais recente foi a de criação do Parque Nacional Marinho do Albardão (ICMBio, 2010), o que determinaria a exclusão total das atividades socioeconômicas dentro dos limites estabelecidos nesta nova UC, gerando uma série de impactos sociais diretos com as comunidades 
e representantes políticos das categorias sociais atingidas (como pescadores artesanais, pescadores industriais, armadores da pesca industrial, rizicultores, silvicultores, parques eólicos, balneários), incrementando os conflitos locais e regionais transescalares, o que promoveria sua radical rejeição e inviabilizaria sua aceitação em quaisquer processos de audiências públicas exigidas, sepultando assim todos os seus esforços de conservação.

Com base nessas informações, o objetivo do presente trabalho foi analisar essas propostas anteriores e desenvolver uma alternativa a estas que possa contribuir para a conservação da biodiversidade e dos recursos pesqueiros, atendo-se aos critérios de Justiça Ambiental na repartição social dos custos e benefícios da apropriação dos espaços e dos recursos naturais, entendida como mais coerente e de maior relevância socioambiental regional.

\section{Metodologia}

Como etapas precedentes à delimitação de novas áreas protegidas na região, foram realizados o reconhecimento e a espacialização de alguns dos principais conflitos socioambientais associados aos seus respectivos potenciais econômicos, explicitando a sobreposição espacial entre os mesmos e os atributos naturais, objetos desta proposta de conservação:

a) Faixa costeira da zona de praia entre o mesolitoral e o infralitoral, com substrato biodetrítico dos Concheiros do Albardão: área de patrimônio paleontológico, com grande deposição de fósseis marinhos (especialmente de tubarões e raias, e macroinvertebrados bentônicos) e continentais (especialmente da megafauna de mamíferos pleistocênicos), em conflito com o trânsito de veículos motorizados 4x4 (usual) e um potencial de mineração de calcário (com locais de mineração já outorgados), podendo ocasionar desestabilização e destruição parcial do depósito paleontológico;

b) Faixa costeira e marinha adjacente, entre o mesolitoral e o infralitoral, abrangendo toda a linha de costa de substrato arenoso e a porção de substrato biodetrítico dos "Concheiros" e dos "Parcéis" do Albardão: área de reprodução e alimentação de teleósteos e elasmobrânquios, toninhas, tartarugas-marinhas e aves marinhas oceânicas (Vooren \& Chiaradia, 1990), em conflito com as pescas artesanal e industrial, podendo causar expressiva mortalidade de raias e tubarões (desovantes e/ou neonatos e juvenis), causada principalmente pela captura incidental por meio da pesca artesanal de arrastão de praia (Klippel et al., 2005; Vooren \& Klippel, 2005b) e industrial (Haimovici et al., 1989; Haimovici \& Mendonça, 1996; Marín et al., 2000; Perez \& Pezzuto, 2006), bem como expressiva mortalidade de tartarugas-marinhas (Barata et al., 1998; Achával et al., 2000; Mäder et al., 2007), aves oceânicas (Barea et al., 2000; Neves et al., 2005; Olmos \& Bugoni, 2006) e toninhas (Moreno et al., 1994; Secchi et al., 1997; Pinedo \& Polacheck, 1999; Secchi et al., 2003), causadas pela captura na pesca industrial (arrasto, emalhe, cerco e/ou espinhel).

Após esta etapa, procedeu-se à revisão crítica de um conjunto de publicações técnicas, científicas e legais disponíveis sobre o tema e a região, estruturando-se um banco de dados e um sistema de informações geográficas, seguida da análise documental das mesmas, com foco na relevância para a conservação da biodiversidade e da ativi- 
dade pesqueira, além dos conflitos entre a gestão ambiental e a gestão pesqueira.

Procurou-se avaliar a percepção social do tema, com entrevistas junto a diferentes atores e setores envolvidos na região. Com base nos critérios da Justiça Ambiental (sensu Acselrad et al., 2004), procedeu-se à elaboração e à discussão de uma matriz dos cenários preditivos (Peres \& Mendiondo, 2004) em termos de impactos socioambientais decorrentes das distintas opções de gestão socioambiental regional.

Para se determinar a validade relativa destas diferentes opções como ferramentas de gestão ambiental e pesqueira, é essencial avaliar o seu desempenho, com indicadores que possam medir os progressos em direção às metas. $\mathrm{Na}$ definição destes indicadores, foi utilizado o quadro conceitual DPSIR [driver-pressão-estado-impacto-resposta] (Kemerich et al., 2014) como um modelo flexível que pode ser adaptado às necessidades de programas específicos para enfatizar diferentes tipos de indicadores. Discutem-se os desdobramentos potenciais de cada uma das opções descritas.

\section{Resultados e discussão}

\subsection{Propostas anteriores de gestão pesqueira e ambiental}

a) No caso das Áreas de Exclusão de Pesca (AEPs), propostas por Vooren \& Klippel (2005b), faz-se necessário um ajuste razoável entre os argumentos ambientais com os socioeconômicos e políticos envolvidos (Figura 2).
Neste caso, os limites propostos da AEP Costa Sul contribuiriam efetivamente para a proteção e a recuperação dos estoques reprodutivos de elasmobrânquios e demais taxa associados, porém, cerceariam a atividade da pesca artesanal em toda a linha de costa, inviabilizando os Modos de Vi$d a$ das comunidades tradicionais remanescentes, desembarcadas (com redes de espera ou "cabos") e embarcadas (com baleeiras a remo), já muito impactadas pela pesca predatória e redução dos estoques. Na primeira categoria, seus agrupamentos vêm decrescendo entre 50 (Klippel et al., 2005) e 20 (Ferreira, 2012), enquanto que para a segunda categoria estima-se que na década de 1980 havia cerca de 60 "parelhas" de baleeiras (com equipes de 20 a 30 pescadores) na costa gaúcha e sete no seu litoral sul (Klippel et al., 2005). Mais recentemente, foi registrada apenas uma parelha no litoral sul (Ferreira, 2012).

De maneira similar, é notório o mérito ecológico da proposta da AEP Plataforma Sul, porém, seus

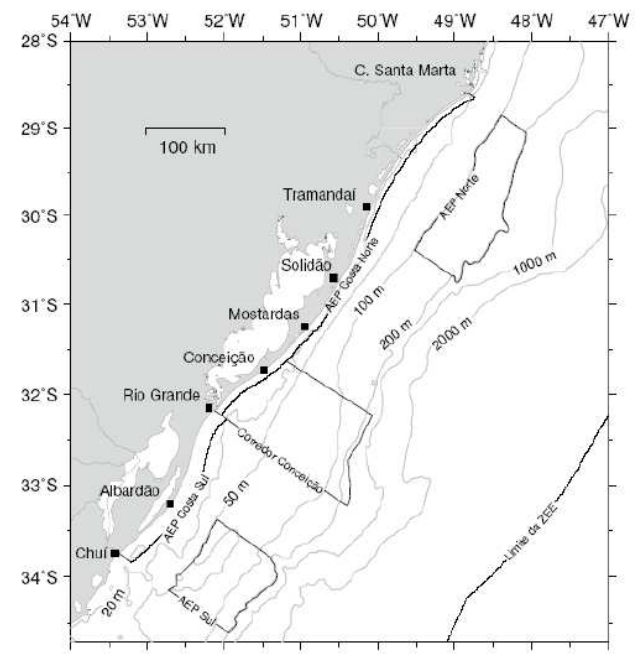

FIGURA 2 - Limites propostos para as Áreas de Exclusão de Pesca. FONTE: Vooren \& Klippel (2005b). 
limites muito amplos e critérios muito restritivos inviabilizariam o exercício da atividade da frota pesqueira industrial naquela que é sua principal área de pesca no sul do Brasil (Perez \& Pezzuto, 2006), com todas as consequências socioeconômicas e políticas decorrentes.

b) Em relação à proposta do Parque Nacional Marinho do Albardão (ICMBio, 2010), enquanto constituinte da categoria de Unidades de Conservação de Proteção Integral do Sistema Nacional de Unidades de Conservação (Brasil, 2000), tal proposição pode não ser a estratégia mais recomendada em relação à expectativa de Desenvolvimento Territorial Sustentável (Vieira et al., 2010) para o contexto socioambiental regional, porque determinaria a exclusão total das atividades socioeconômicas dentro dos seus limites, gerando uma série de impactos sociais diretos com as comunidades atingidas, especialmente os pescadores artesanais, bem como com os pescadores da frota industrial, armadores e industriais da pesca.

Esta supressão absoluta dos direitos de acesso aos recursos pesqueiros em grande parte das áreas de exercício da atividade incrementaria os conflitos transescalares, o que promoveria sua radical rejeição sociopolítica e inviabilizaria sua aceitação, sepultando a potencial eficácia da Unidade de Conservação proposta em prol da conservação ambiental. A perspectiva da efetiva conservação dos espaços e dos recursos naturais da região necessita ter aderência social e comunitária, quando os atores sociais forem envolvidos no processo decisório e tiverem atendidas suas necessidades e expectativas, tornando-se beneficiários e não excluídos do mesmo. Entendemos que muito mais eficaz do que tentar proibir toda a atividade pesqueira na área (inviável técnica e politicamente) seria buscar a implantação de mecanismos de corresponsabilidade na conservação dos estoques reprodutivos, com um sistema de manejo e monitoramento integrados, envolvendo as agências ambientais e o setor pesqueiro.

\subsection{Alternativas de gestão socioambiental pesqueira}

Uma contribuição significativa para as iniciativas de gestão socioambiental da pesca regional remete à primeira proposta, concebida em termos consensuais e integradores, de criação de Áreas de Exclusão de Pesca (AEPs) marinhas no Rio Grande do Sul (Peres et al., 2007), por meio de processo de gestão participativa pelos pescadores artesanais de 11 comunidades no Litoral Norte RS. Este caso é representativo das possibilidades de manejo eficaz, ecológica e socialmente, pela concepção, pelo desenho, pela implantação e fiscalização conjuntas das áreas de exclusão de pesca, pois os pescadores reconhecem e apontam quais as áreas que devem ter a atividade excluída e se comprometem a respeitar e fazer respeitar os limites espaço-temporais acordados. Além de diminuir drasticamente o esforço de pesca, a implantação destas AEPs seria um mecanismo importante de proteção de áreas críticas para a conservação da biodiversidade.

Esta iniciativa valoriza o Conhecimento Ecológico Tradicional (Berkes, 1999; Neis \& Felt, 2000) dos pescadores, por entender que os mesmos detêm grande conhecimento, aplicável e necessário nos estudos ictiológicos, na biologia pesqueira, no manejo da pesca, na biologia da conservação marinha, no desenho e no manejo de Áreas Marinhas Protegidas (Gerhardinger et al., 2009).

As aproximações ecológicas à gestão ambiental devem ser reforçadas por uma compreensão da 
dinâmica política de como estas se relacionam às ações humanas (Cumming \& Peterson, s.d.). Procurando incorporar tais contribuições na proposição de medidas de gestão socioambiental e pesqueira para a região do Albardão, recomenda-se a implantação de um Mosaico de Unidades de Conservação (MMA, s.d.), resguardando a estrutura e a função ecológica dos ecossistemas, a integridade dos modos de vida das comunidades tradicionais de pesca artesanal e a viabilidade econômica da pesca industrial atuante na região. De modo a disponibilizar alternativas para distintas escalas de abrangência e sistemas de gestão, na seleção e no estudo de viabilidade para a delimitação das melhores opções deste Mosaico de UCs, foram desenvolvidos dois modelos:

a) Mosaico Compacto: RDS Lagoas Mirim-Mangueira, e REVIS Concheiros e Parcéis do Albardão (Figura 3).

b) Mosaico Expandido: REVIS Arroio Del Rey, RDS Lagoas Mirim-Mangueira, REVIS Concheiros do Albardão, e AMP Parcéis do Albardão (Figura 4).

\subsection{Unidades de Conservação do Mosaico de UCs}

RDS Lagoas Mirim-Mangueira: na delimitação proposta para esta Reserva de Desenvolvimento Sustentável das Lagoas Mirim e Mangueira, foram consideradas as áreas tradicionais de pesca e residência das comunidades de pesca artesanal em ambas as lagoas, abrangendo os seus territórios mais amplos, envolvendo as suas rotas de navegação e deslocamento. Também foram consideradas as áreas vitais para as lavouras rizícolas circundantes (os pontos de captação de água para as mesmas), assim como a necessidade de garantir a progressiva substituição do modo de produção agroquímico nestes cultivos, especialmente nas proximidades dos corpos d'água e no controle dos efluentes destas lavouras.

REVIS Arroio Del Rey: o Refúgio da Vida Silvestre do Arroio Del Rey visa assegurar a conservação dos últimos remanescentes dos grandes sistemas de banhados e dos adjacentes palmares de butiá (Butia capitata e $B$. odorata), ambos, em grande parte, destruídos pela expansão da lavoura rizícola irrigada e pela pecuária extensiva. A área proposta configura-se como um expressivo delta meandrante interno, que deságua na Lagoa Mirim, em cujas margens e ilhas se localizam significativas áreas para reprodução das principais espécies de peixes dulciaquícolas regionais (Garcia et al., 2006), dos quais dependem os pescadores artesanais lagunares (Pieve et al., 2009), além de refúgios, grandes áreas de alimentação e ninhais de anfíbios, répteis, mamíferos silvestres e aves residentes e migratórias (Guadagnin et al., 2005).

REVIS Concheiros do Albardão: esta face submersa dos Concheiros do Albardão constitui-se numa estrutura tridimensional biodetrítica, que fornece o suporte físico para a instalação de toda uma ampla e diversificada comunidade de algas e macroinvertebrados bentônicos, com um intrincado sistema de cânions, que garante a recirculação de nutrientes. Entende-se como necessária a proteção de uma área representativa da distribuição e/ou reprodução das espécies ameaçadas, optando-se por um traçado que envolve o alinhamento dos Faróis do Sarita e do Albardão (seus vértices NE e SE, no mar), os vértices NW (a base do Farol do Sarita) e SW (entre a base dos Concheiros e a isóbata de 20 metros, na face submersa dos Concheiros). Este REVIS também asseguraria a conservação dos patrimônios geológico, paleontológico e cultural associados aos campos de dunas eólicas e depósitos fossilíferos em sua face praial emersa e submersa (Lopes et al., 2007). 


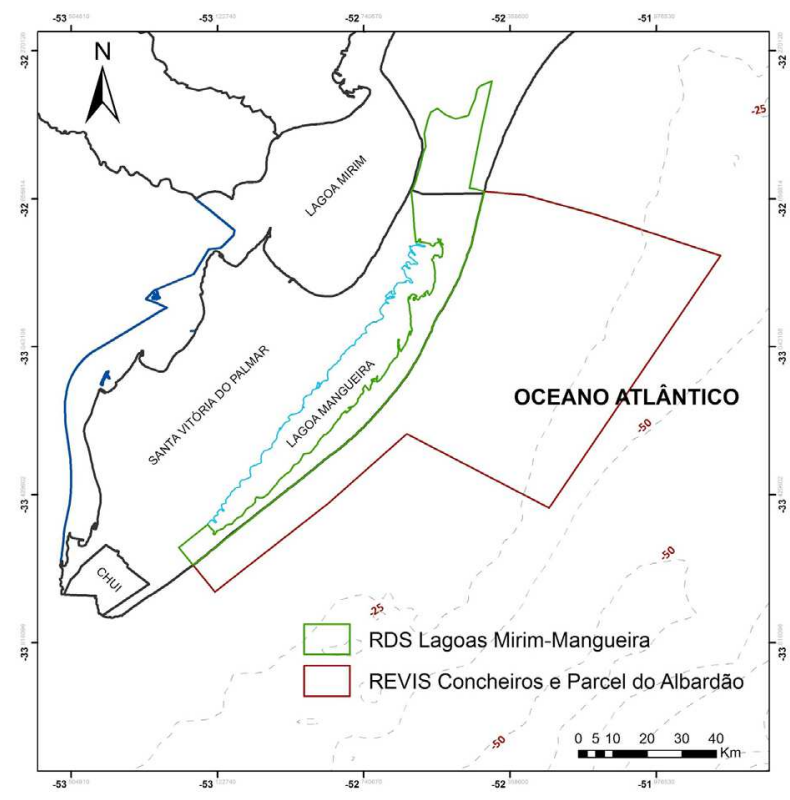

FIGURA 3 - Proposta de Mosaico Compacto de Unidades de Conservação do Albardão, RS - RDS LMM: Reserva de Desenvolvimento Sustentável das Lagoas Mirim e Mangueira; REVIS CPA: Refúgio da Vida Silvestre dos Concheiros e Parcéis do Albardão.

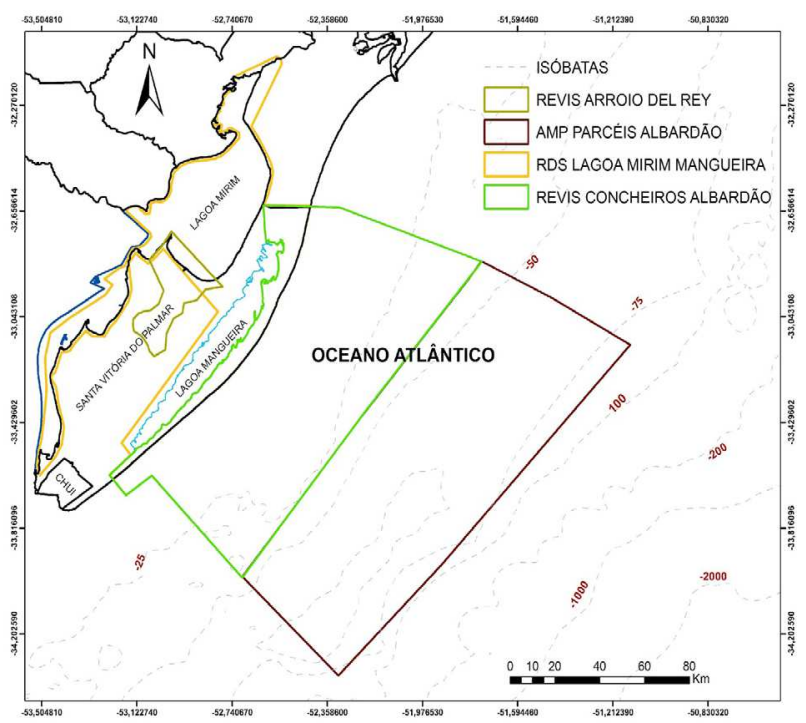

FIGURA 4 - Proposta de Mosaico Expandido de Unidades de Conservação do Albardão, RS - RDS LMM: Reserva de Desenvolvimento Sustentável das Lagoas Mirim e Mangueira; REVIS ADR: Refúgio da Vida Silvestre do Arroio Del Rey; REVIS CA: Refúgio da Vida Silvestre dos Concheiros do Albardão; $A M P$ PA: Área Marinha Protegida dos Parcéis do Albardão. 
REVIS CPA - Concheiros e Parcéis do Albardão: esta proposta condensada de REVIS integra as duas áreas marinhas mais significativas do Albardão - Zm002 e Zm003 (MMA, 2007): habitats de substrato consolidado (cascalho biodetrítico) intercalado com fácies areno-lamosas como áreas muito significativas para abrigo e agregação reprodutiva de várias espécies de peixes; área de concentração e alimentação de tartarugas-marinhas e aves oceânicas ameaçadas pela pesca; captura incidental de aves, tartarugas e mamíferos marinhos. Área de intensa atividade da frota pesqueira industrial, nas suas diversas categorias e artes de pesca.

AMP Parcéis do Albardão: a proposição de uma Área Marinha Protegida-AMP segue as recomendações internacionais da IUCN para o manejo responsável no ambiente marinho (Kelleher, 1999); sua localização foi estabelecida de modo a fornecer a possibilidade de um corredor de deslocamento para os cardumes, livre da pressão de pesca pela frota industrial, que garanta o sucesso reprodutivo das espécies ameaçadas. O seu reconhecimento poderia ser viabilizado por meio da integração dos instrumentos legais de uma APA - Área de Proteção Ambiental - e dos instrumentos técnicos de uma Área de Exclusão de Pesca.

A sua delimitação espacial buscou uma redução do ônus à atividade de pesca industrial e ao equilíbrio do custo relativo deste esforço de gestão dos recursos pesqueiros entre os principais usuários da frota industrial, distribuindo-se o perímetro proposto de modo a recobrir parcialmente as áreas de pesca de diferentes modalidades (arrasto, espinhel, emalhe, cerco, covos), assim evitando-se penalizar exclusivamente um(a) ou outro(a) arte/processo de pesca. O processo de gestão desta AMP poderia adotar os critérios do manejo adaptativo, por meio do qual, com o monitoramento científico dos resultados obtidos com a exclusão da atividade pesqueira, os limites espaço-temporais da mesma poderiam ser progressivamente ajustados às respostas ambientais dos procedimentos em curso (expandindo, contraindo e/ou deslocando os limites físicos e/ou os períodos de exclusão de pesca).

\subsection{Cenários preditivos}

Os cenários não são previsões, projeções ou predições, são caminhos críticos sobre os futuros possíveis e visam reduzir as incertezas na tomada de decisões. O processo de construção de cenários é baseado na formulação de questões-chave, sugerindo respostas plausíveis e na orientação às ações julgadas mais adequadas. Ao oferecer a introspecção nas incertezas e nas consequências das ações atuais, os cenários tornam o processo de tomada de decisão mais informado e racional. Adicionar tal foco utilizado na construção de cenários é um alvo preliminar na avaliação dos modelos de gestão aplicáveis aos ecossistemas (Raskin, 2005).

$\mathrm{Na}$ avaliação dos potenciais impactos socioambientais decorrentes das distintas opções de gestão socioambiental regional, foi elaborada uma matriz dos cenários preditivos, em termos de [(a: sem a criação da UC); (b: com a criação da UC; (b1: como Parque Nacional); (b2: como Mosaico de UCs); (b2.1: Mosaico expandido); (b2.2: Mosaico compacto)] (Figura 5).

Tais resultados condensam os potenciais e as restrições socioambientais implícitos em cada uma das opções de gestão para a região, focalizando os principais setores e atividades envolvidos na disputa sobre os espaços e os recursos naturais, ou seja, a 
conservação da biodiversidade e da ictiofauna associada, bem como o exercício das pescas artesanal e industrial. Esta análise comparada constitui-se em instrumento de apoio à decisão dos gestores, por explicitar o custo-benefício socioambiental de cada uma das opções investigadas. Como consequência do registro histórico dos fracassos da gestão pesqueira centralizada, existe a necessidade de mudanças na estrutura de governança (Thé \& Ruffino, 2009). Necessita-se de uma parceria mais dinâmica, usando as capacidades e os interesses dos pescadores e das comunidades locais, complementados pela capacidade de o Estado possibilitar uma legislação de apoio, fiscalização e outras formas de amparo. Essa abordagem de gestão pesqueira exige um afastamento da forma de gestão centralizada e de cima para baixo, buscando uma nova estratégia na qual os gestores pesqueiros e pescadores possam gerir as pescarias em conjunto: a gestão compartilhada (Jentoft, 1989; Pinkerton, 1989; Berkes et al., 1991). A concepção de "Gestão Compartilhada" preconiza o compartilhamento do poder de decisão e da responsabilidade na gestão dos espaços e dos recursos naturais entre o Estado e os grupos que se utilizam dos mesmos (Pinkerton, 1989). Existem diversas experiências documentadas da adoção desta modalidade de gestão, especialmente envolvendo comunidades e recursos pesqueiros (Jentof \& McCay, 1995 apud Berkes et al., 2006).

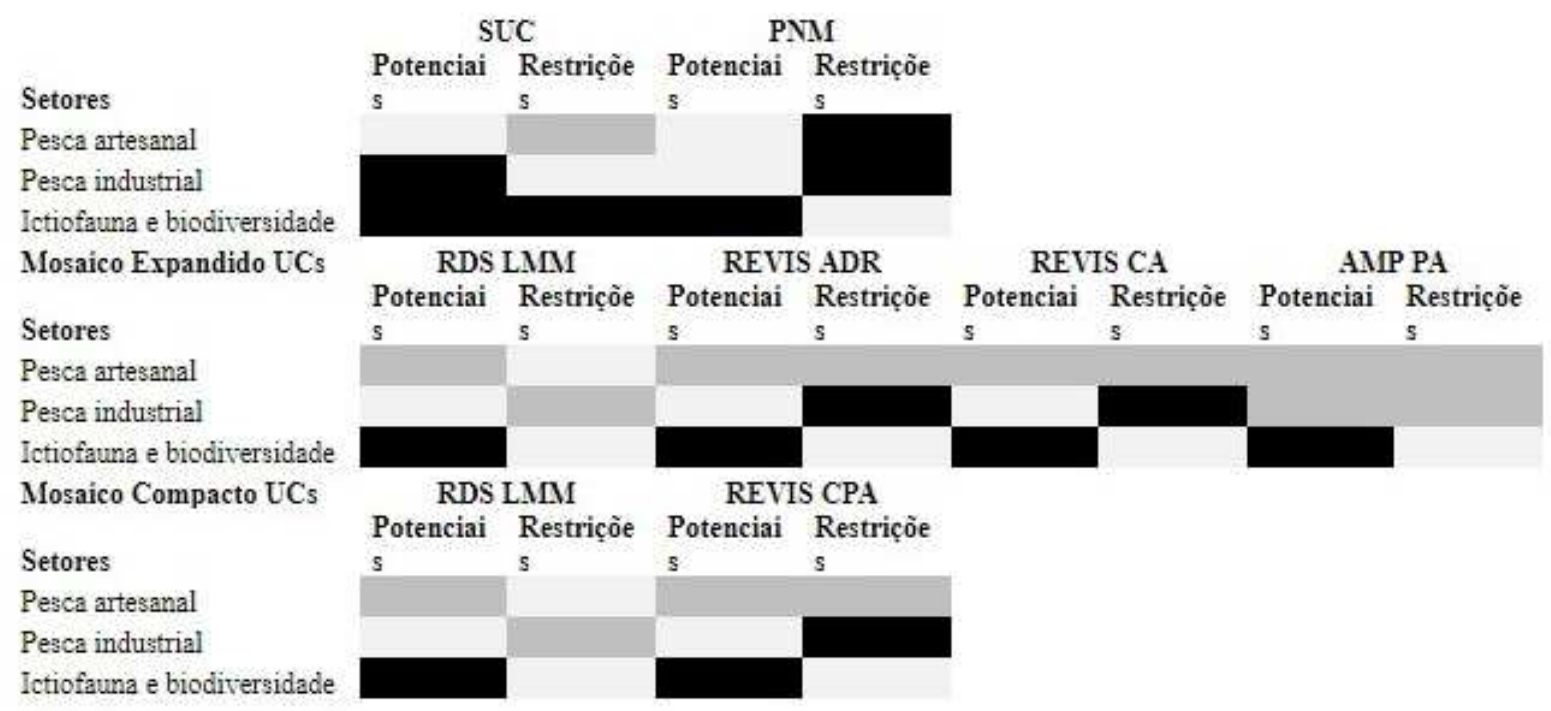

FIGURA 5 - Matriz de cenários preditivos da criação de UC na região do Albardão, RS. SUC: sem Unidade de Conservação; PNM: Parque Nacional Marinho do Albardão; RDS LMM: Reserva de Desenvolvimento Sustentável das Lagoas Mirim e Mangueira; REVIS ADR: Refúgio da Vida Silvestre Arroio Del Rey; REVIS CPA: Refúgio da Vida Silvestre dos Concheiros e Parcéis do Albardão; REVIS CA: Refúgio da Vida Silvestre dos Concheiros do Albardão; AMP PA: Área Marinha Protegida dos Parcéis do Albardão.
Legenda

$$
\text { Alto }
$$$$
\text { Médio }
$$
Baixo 


\section{Conclusão}

Considerando-se a complexidade socioambiental da região do Albardão, em função de sua representatividade para a conservação da biodiversidade regional, assim como seu significado socioeconômico e cultural para os protagonistas e as atividades das pescas artesanal e industrial, recomenda-se a adoção de um modelo de gestão costeira integrada, por meio da implantação de um amplo Mosaico de Unidades de Conservação, resguardando a estrutura e a função ecológica dos ecossistemas, promovendo a progressiva recuperação das espécies e dos estoques ameaçados, a conservação da integridade dos modos de vida das comunidades tradicionais de pesca artesanal e a viabilidade econômica da pesca industrial.

As análises desenvolvidas revelaram que a proposta do Mosaico Expandido exibe melhor ajuste dos custos e benefícios socioambientais, incrementa os potenciais para conservação da biodiversidade (pela abrangência e conectividade ecossistêmica entre a bacia hidrográfica e a zona costeiro-marinha) e minimiza as restrições espaço-temporais para a atividade pesqueira (equalizando-as entre as diversas categorias de atores sociais, em função de suas distintas escalas de operações, resiliência, capitais tecnológico e econômico). Embora a área total projetada para este mosaico seja quase tão ampla como a proposta para a criação do Parque Nacional Marinho, entendemos que a mesma possa ser muito mais eficaz justamente por incorporar a complexidade socioambiental a seu favor, ao buscar a coalizão de interesses e atividades socioeconômicas regionais, em diferentes escalas e com diferentes e complementares mecanismos de regulação, segundo as diversas categorias de UCs nele contidas.

Tal proposição contribuiria também para a estruturação de um Corredor Ecológico, conectando estas Áreas Marinhas Protegidas do extremo sul do Brasil com a porção setentrional do Sistema de Áreas Protegidas do Uruguai, através de sua AMP Cerro Verde (Lopez et al., 2011), viabilizando o manejo binacional dos recursos naturais compartilhados, protegendo muitas das espécies-bandeiras e estoques pesqueiros notadamente migratórios ou com grandes deslocamentos sazonais que se utilizam desta região transfronteiriça.

Em uma região tão remota e escassamente povoada, as dificuldades para a fiscalização ambiental centralizada são inequívocas; porém, estas podem ser amenizadas quando são preservados os interesses socioeconômicos, seja pela continuidade dos direitos de acesso aos recursos pesqueiros, seja pela percepção da manutenção da capacidade de suporte dos mesmos e da consequente sustentabilidade financeira do setor. Neste sentido, o sucesso de tal proposta (e, com ela, a conservação da biodiversidade regional) ratifica a indissociabilidade entre os princípios e métodos da gestão ambiental com aqueles da educação ambiental (Loureiro \& Cunha, 2008; Quintas et al., 2006; Quintas, s.d.).

\section{Referências}

Achával, F.; Marín, Y. H.; Barea, L. C. Captura incidental de tortugas con palangre pelágico oceánico en el Atlántico Sudoccidental. In: Arena, G.; Rey, M. (Orgs.). Captura de grandes peces pelágicos (pez espada y atunes) en el atlántico sudoccidental, y su interacción con otras poblaciones. Proyecto Uru/92/003. Montevideo (Uruguay): Instituto Nacional de Pesca - Programa de las Naciones Unidas para 
el Desarrollo, 2000. p. 83-88.

Acselrad, H.; Herculano, S.; Pádua, J. A. Justiça Ambiental e cidadania. Rio de Janeiro: Relume Dumará, 2004.

Arnestein, S. Uma escada da participação cidadã. Revista da Associação Brasileira para a Promoção da Participação, 2(2), 4-13, 2002.

Attisano, K. K.; Niencheski, L. F. H.; Milani, I. C. B.; Machado, C. S.; Milani, M. R.; Zarzur, S.; Andrade, C. F. F. Evidences of Continental Groundwater Inputs to the Shelf Zone in Albardão, RS, Brazil. Brazilian Journal of Oceanography, 56(3), 189-200, 2008. Disponível em: <http:// www.scielo.br/pdf/bjoce/v56n3/v56n3a04.pdf>.

Barata, P. C. R.; Gallo, B. M. G.; Santos, S.; Azevedo, V. G.; Kotas, J. E. Captura acidental da tartaruga marinha Caretta caretta na pesca de espinhel de superfície na ZEE brasileira e em águas internacionais. In: Proceedings of the $11^{\text {th }}$ Semana Nacional de Oceanografia. Rio Grande, RS. FURG - Universidade Federal do Rio Grande, 1998.

Barea, L.; Loinaz, L.; Marin, Y.; Ríos, C.; Saralegui, A.; Stagi, A.; Vaz-Ferreira, R.; Wilson, N. Mortalidad de albatros y otras aves marinas producida en aguas uruguayas por la pesca de atunes con palangre. In: Arena, G.; Rey, M. (Orgs.). Captura de grandes peces pelágicos (pez espada y atunes) en el atlántico sudoccidental, y su interacción con otras poblaciones. Proyecto Uru/92/003. Montevideo (Uruguay): Instituto Nacional de Pesca - Programa de las Naciones Unidas para el Desarrollo, 2000. p. 71-81.

Berkes, F. Sacred Ecology: Traditional ecological knowledge and resource management. Philadelphia: Taylor and Francis, 1999.

Berkes, F.; George, P. J.; Preston, R. J. Co-management: The evolution of the theory and practice of joint administration of living resources. Program for Technology Assessment in Subarctic Ontario, McMaster University, 1991.

Berkes, F.; Mahon, R.; McConey, P.; Pollnac, R.; Pomeroy, R. Gestão da pesca de pequena escala: diretrizes e métodos alternativos. Rio Grande: Editora da FURG, 2006.

Brasil. Lei $n^{\circ}$ 9.985, de 18 de julho de 2000. Institui o SNUC - Sistema Nacional de Unidades de Conservação. Brasília: DOU de 19/07/2000.
Bugoni, L.; Mancini, P. L.; Monteiro, D. S.; Nascimento, L.; Neves, T. S. Seabird bycatch in the Brazilian pelagic longline fishery and a review of capture rates in the southwestern Atlantic Ocean. Endangered Species Research, 5, 137-147, 2008. doi: $10.3354 /$ esr00115

Bugoni, L.; D’Alba, L.; Furness, R. W. Marine habitat use of wintering spectacled petrels Procellaria conspicillata, and overlap with longline fishery. Marine Ecology Progress Series, 374, 273-285, 2009. doi: 10.3354/meps07750

Chao, L. N.; Pereira, L. E.; Vieira, J. P. Estuarine fish community of the Patos Lagoon, Brazil: a baseline study. In: Yánez-Arancibia, A. (Org.). Fish Community Ecology in Estuaries and Coastal Lagoons: Towards an Ecosystem Integration. Ciudad de México: UNAM Press, 1985. p. 429-450.

Cumming, G.; Peterson, G. Ecology in Global Scenarios. In: Carpenter, S. R.; Pingali, P. L.; Bennett, E. M.; Zurek, M. (Orgs.). Ecosystems and Human Well-Being: Scenarios. Island Press, 2005. p. 45-71.

Ferreira, E. C.; Muelbert, M.; Secchi, E. R. Distribuição espaçotemporal das capturas acidentais de toninhas (Pontoporia blainvillei) em redes de emalhe e dos encalhes ao longo da costa sul do Rio Grande do Sul, Brasil. Atlântica, 32, 183-197, 2010. Disponível em: <http://repositorio.furg. $\mathrm{br} /$ handle/1/3578>.

Ferreira, W. Diagnóstico pesqueiro e dos potenciais econômicos vinculados aos ambientes costeiros. Relatório de Consultoria Ambiental - Projeto BRA/08/023 [análise do projeto de criação do Parque Nacional Marinho do Albardão, RS]. ICMBio - Instituto Chico Mendes de Conservação da Biodiversidade / PNUD - Programa das Nações Unidas para o Desenvolvimento, Março de 2012. 140 p.

Garcez, D. S.; Sánchez-Botero, J. I. Comunidades de pescadores artesanais no estado do Rio Grande do Sul, Brasil. Atlântica, 27(1), 17-29, 2005.

Garcia, C. A. E. Características Hidrográficas. In: Seeliger, U.; Odebrecht, C.; Castello, J. P. Os Ecossistemas Costeiro e Marinho do Extremo Sul do Brasil. Rio Grande, RS: Ecoscientia, 1998. p. 18-21.

Garcia, A. M.; Bemvenuti, M. A.; Vieira, J. P.; Marques, D. M. L. M.; Burns, M. D. M.; Moresco, A.; Condini, M. 
V. L. Checklist comparison and dominance patterns of the fish fauna at Taim Wetland, South Brazil. Neotropical Ichthyology, 4(2), 261-268, 2006. doi: 10.1590/S167962252006000200012

Gerhardinger, L. C.; Godoy, E. S.; Jones, P. J. S. Local ecological knowledge and the management of marine protected areas in Brazil. Ocean \& Coastal Management, 52(3-4), 154-165, 2009. doi: 10.1016/j.ocecoaman.2008.12.007

Guadagnin, L. D.; Peter, A. S.; Perello, L. F. C.; Maltchik, L. Spatial and Temporal Patterns of Waterbird Assemblages in Fragmented Wetlands of Southern Brazil. Waterbirds, 28(3), 261-272, 2005. doi: 10.1675/1524-4695(2005)028[0261:SATPOW]2.0.CO;2

Haimovici, M.; Pereira, S. D.; Vieira, P. C. La pesca demersal en el sur de Brasil en el período 1975-1985. Frente Maritimo, 5, 151-163, 1989.

Haimovici, M.; Mendonça, J. T. Descartes da fauna acompanhante na pesca de arrasto e tangones dirigida a linguados e camarões na plataforma continental do sul do Brasil. Atlântida, 18, 161-177, 1996.

Haimovici, M.; Martins, A. S.; Vieira, P. C. Distribuição e abundância de peixes teleósteos demersais sobre a plataforma continental do Sul do Brasil. Revista Brasileira de Biologia, 56(1), 27-50, 1996.

Iclarm. Dissemination and evaluation of genetically improved tilapia species in Asia. Final Report. International Centre for Living Aquatic Resources Management, Manila, Philippines, 1998. p. 35-45.

ICMBio - Instituto Chico Mendes de Conservação da Biodiversidade. Projeto para conservação da biodiversidade e promoção do desenvolvimento socioambiental: Projeto PNUD BRA/08/023, Código BRA/08/023. Brasília, 2010.

Jentoft, S. Fisheries co-management: delegating government responsibility to fishermen's organizations. Marine Policy, 13(2), 137-154, 1989. doi: 10.1016/0308-597X(89)90004-3

Jentoft, S.; McCay, B. J. User participation in fisheries management: lessons drawn from international experience. Marine Policy, 19, 227-246, 1995.

Kalikoski, D. C.; Seixas, C. S.; Almudi, T. Gestão compartilhada e comunitária da pesca no Brasil: avanços e desafios.
Ambiente \& Sociedade, XII(1), 151-172, 2009.

Kalikoski, D. C.; Rocha, R. D.; Vasconcellos, M. C. Importância do conhecimento ecológico tradicional na gestão da pesca artesanal no estuário da Lagoa dos Patos, extremo sul do Brasil. Ambiente \& Educação, 11(1), 87-118, 2006. Disponível em: $<$ https://www.seer.furg.br/ambeduc/article/ view/770>.

Kelleher, G. (Org.). Guidelines for Marine Protected Areas. Cambridge University Press, 1999.

Kemerich, P. D. C.; Ritter, L. G.; Borba, W. F. Indicadores de Sustentabilidade Ambiental: métodos e aplicações. REMOA - Revista Monografias Ambientais (Edição Especial LPMA/ UFSM), 13(5), 3723-3736, 2014. Disponível em: <https:// periodicos.ufsm.br/remoa/article/viewFile/14411/pdf $>$.

Klippel, S.; Peres, M. B.; Carvalho, R. V. Modelo alternativo para avaliação e gestão da pesca artesanal no entorno da Estação Ecológica do Taim, Rio Grande do Sul, Brasil: sustentabilidade da pesca e conservação da biodiversidade. In: Anais do IX Congresso da Associação Brasileira de Estudos do Quaternário e do II Congresso do Quaternário de Países de Línguas Ibéricas, Recife, 2003. Disponível em: $<$ http://www.abequa.org.br/trabalhos/recursos_119.pdf $>$.

Klippel, S.; Peres, M. B.; Vooren, C. M.; Lamónaca, A. F. A pesca artesanal na costa da Plataforma Sul. In: Vooren, C. M.; Klippel, S. (Orgs.). Ações para a conservação de tubarões e raias no Sul do Brasil. Porto Alegre: Igaré Editora, 2005. p. 179-197.

Lopes, R. P.; Ugri, A.; Buchmann, F. S. C. Dunas do Albardão, RS: bela paisagem eólica no extremo sul da costa brasileira. Proposta de Sítio Geológico ou Paleontológico do Brasil a ser preservado como Patrimônio Natural da Humanidade. In: SIGEP 03: Comissão Brasileira de Sítios Geológicos e Paleobiológicos, Setembro/2007. Disponível em: $<$ http://www.unb.br/ig/sigep/propostas/Dunas_do_Albardao_RS.htm>.

Lopez, M.; Fallabrino, A.; Brazeiro, A. Incorporación del Área Costero-Marina "Cerro Verde" a el Sistema Nacional de Áreas Protegidas, Uruguay. 2011. Disponível em: $<$ www.karumbe.org.uy $>$.

Loureiro, C. F. B.; Cunha, C. C. Educação ambiental e gestão participativa de unidades de conservação: elementos 
para se pensar a sustentabilidade democrática. Ambiente \& Sociedade, XI(2), 237-253, 2008.

Mäder, A.; Sander, M.; Casa Jr., G. E; Altenhofen, R. J; Anjos, C. S.; Leão, P. P.; Bicca, L. B. Impacto antrópico em tartarugas marinhas no sul do Brasil. In: Anais do XII Congresso Latino-Americano de Ciências do Mar - XII COLACMAR. Florianópolis, 15-19 de abril de 2007.

Mancini, P. L.; Bugoni, L.; Neves, T. S.; Monteiro, D. S.; Estima, S. C. The effect of light toriline on seabird by-catch and fish catch rates in the pelagic longline fishery off southern Brazil. Collective Volume of Scientific Papers. International Commission for the Conservation of Atlantic Tunas, 64, 2499-2507, 2009.

Marín, Y. H.; Brum, F.; Barea, L. C.; Chocca, J. F. Captura incidental y su aprovechamiento en la pesquería de pez espada con palangre pelágico en el Atlántico Sudoccidental. In: Arena, G.; Rey, M. (Orgs.). Captura de grandes peces pelágicos (pez espada y atunes) en el atlántico sudoccidental, y su interacción con otras poblaciones. Proyecto Uru/92/003. Montevideo (Uruguay): Instituto Nacional de Pesca - Programa de las Naciones Unidas para el Desarrollo, 2000.

MMA - Ministério do Meio Ambiente. Áreas Prioritárias para Conservação, Uso Sustentável e Repartição dos Benefícios da Biodiversidade Brasileira. Portaria MMA n ${ }^{\circ}$ 9, de 23 de janeiro de 2007. Biodiversidade, n. 31. 2007. Brasília: DOU de 24/01/2007.

MMA - Ministério do Meio Ambiente. Mosaicos de Unidades de Conservação. Sistema Nacional de Unidades de Conservação. Brasília, s.d. Disponível em: <http://www. mma.gov.br/sitio/index.php?ido=conteudo.monta\&idEstrutura $=240 \&$ idConteudo $=10099 \&$ idMenu $=11707>$.

Moreno, I. B.; Ott, P. H.; Danilewicz, D. Análise preliminar do impacto da pesca artesanal costeira sobre Pontoporia blainvillei no litoral norte do Rio Grande do Sul, sul do Brasil. In: Pinedo, M. C.; Barreto, A. (Orgs.). Proceedings of the Second Workshop for the Research Coordination and Conservation of the Franciscana (Pontoporia blainvillei) in the Southwestern Atlantic. Florianópolis, 1994. p. 31-41.

Moreno, I. B.; Tavares, M.; Danilewicz, D.; Ott, P. H.; Machado, R. Descrição da pesca costeira de média escala no litoral norte do Rio Grande do Sul: comunidades pesqueiras de Imbé/Tramandaí e Passo de Torres/Torres. Boletim do Instituto de Pesca, 35(1), 129-140, 2009.

Neis, B.; Felt, L. Finding our sea legs: linking fishery people and their knowledge with science and management. Institute of Social and Economic Research. St Johns, 2000.

Neves, T. S.; Bugoni, L.; Monteiro, D. S.; Nascimento, L.; Peppes, F. V. Seabirds abundance and bycatch on Brazilian longline fishing fleet. CCAMLR/WG-FSA-05/67, 2005.

Niencheski, L. F.; Windom, H. L. Nutrient flux and budget in Patos Lagoon estuary. The Science of the Environment, 149, 53-60, 1994.

Olmos, F.; Bugoni, L. Agregações de aves marinhas associadas à pesca de espinhel-de-fundo na região sudeste-sul do Brasil. In: Neves, T.; Bugoni, L.; Rossi-Wongtschowski, C. L. B. Aves oceânicas e suas interações com a pesca na região sudeste-sul do Brasil. São Paulo, I.O. USP, 2006. p. 69-81.

Peres, R. B.; Mendiondo, E. M. Desenvolvimento de cenários de recuperação como instrumento ao planejamento ambiental e urbano: bases conceituais e experiências práticas. In: Anais do Seminário NEUR/CEAM, UnB "A questão Ambiental e Urbana: Experiências e Perspectivas", Brasília, 2004.

Perez, J. A. A.; Pezzuto, P. R. A pesca de arrasto de talude do Sudeste e Sul do Brasil: tendências da frota nacional entre 2001 e 2003. Boletim do Instituto de Pesca, 32, 127-150, 2006. Disponível em: <ftp://ftp.sp.gov.br/ftppesca/32_2_127-150.pdf>.

Peres, M. B.; Klippel, S.; Vianna, M. A. C. Áreas de exclusão de pesca propostas no processo de gestão participativa da pesca artesanal no Litoral Norte do Rio Grande do Sul: um relato de experiência. In: Prates, A. P.; Blanc, D. (Orgs.). Areas Aquáticas Protegidas como instrumento de Gestão Pesqueira. Brasília, 2007. p. 141-158.

Pieve, S. M. N.; Kubo, R. R.; Souza, G. C. Pescadores artesanais da Lagoa Mirim: etnoecologia e resiliência. Brasília, DF: MDA - Ministério do Desenvolvimento Agrário, 2009.

Pinedo, M. C.; Polacheck, T. Trends in franciscana (Pontoporia blainvillei) stranding rates in Rio Grande do Sul, 
Southern Brazil (1979-1998). Journal of Cetacean Research and Management, 1, 179-189, 1999.

Pinkerton, E. (Org.). Co-operative management of local fisheries: new directions for improved management and community development. Vancouver: University of British Columbia Press, 1989.

Piola, A. R.; Campos, E. J.; Möller, Jr., O. O.; Charo, M.; Martinez, C. The subtropical shelf front of Eastern South America. Journal of Geophysical Research, 105(3), 65656578, 2000.

Quintas, J. S. Educação no Processo de Gestão Ambiental Pública: a construção do ato pedagógico. Brasília: ICMBIO - Instituto Chico Mendes de Conservação da Biodiversidade, s.d. Disponível em: <http://www.icmbio. gov.br/educacaoambiental/images/stories/biblioteca/educacao_ambiental/EDUCACAO_NO_PROCESSO_DE_GESTAO_AMBIENTAL_PUBLICA.pdf $>$.

Quintas, J. S.; Gomes, P. M.; Uema, E. E. Pensando e praticando a Educação no Processo de Gestão Ambiental: uma concepção pedagógica e metodológica para a prática da educação ambiental no licenciamento. Brasília: IBAMA - Instituto Brasileiro do Meio Ambiente e dos Recursos Naturais Renováveis, 2006. Disponível em: <http://www. ibama.gov.br/sophia/cnia/livros/pensandoepraticando2005digital.pdf $>$.

Raskin, P.D. Global scenarios: background review for the Millennium Ecosystem Assessment. Ecosystems, 8, 133142, 2005. doi: 10.1007/s10021-004-0074-2

Rossi-Wongtschowski, C. L. D. B.; Madureira, L. S. P. $O$ ambiente oceanográfico da plataforma continental e do talude na região Sudeste-Sul do Brasil. São Paulo: Editora da Universidade de São Paulo, 2006.

Schroeder, F. A.; Castello, J. P. "Cardume associado": Nova modalidade de pesca de atuns no sul do Brasil - descrição e comparação. Pan-American Journal of Aquatic Sciences, 2(1), 66-74, 2007.

Secchi, E. R.; Zerbini, A. N.; Bassol, M.; Dalla Rosa, L.; Moller, L. M.; Rocha-Campos, C. C. Mortality of franciscanas, Pontoporia blainvillei, in coastal gillnetting in southern Brazil. Report of the International Whaling Commission, 47, 653-658, 1997.
Secchi, E. R.; Ott, P. H.; Danilewicz, D. Effects of fishing bycatch and the conservation status of the franciscana dolphin, Pontoporia blainvillei. In: Gales, N.; Hindell, M.; Kirkwood, R. (Orgs.). Marine Mammals: Fisheries, Tourism and Management Issues. Collingwood (Austrália), CSIRO Publishing, 2003. p. 174-191.

Seixas, C. S; Kalikoski, D. C. Relatório de atividades do workshop "Gestão Compartilhada de Recursos Pesqueiros no Brasil”. Tamandaré, PE: CEPENE, maio 2006.

Seixas, C. S.; Kalikoski, D. C.; Almudi, T.; Batista, V. S.; Costa, A. L.; Diogo, H. L.; Ferreira, B. P.; Futemma, C. R. T.; Moura, R. L; Ruffino, M. L.; Salles, R.; Thé, A. P. G. Gestão compartilhada do uso de recursos pesqueiros no Brasil: elementos para um programa nacional. Ambiente \& Sociedade, XIV(1), 23-44, 2011.

Silva Jr., C. L.; Kampel, M.; Araújo, C. E. S.; Stech, J. L. Observação da penetração do ramo costeiro da Corrente das Malvinas na costa sul-sudeste do Brasil a partir de imagens AVHRR. In: Anais do VIII Simpósio Brasileiro de Sensoriamento Remoto. Salvador, 14-19 de abril de 1996.

Souza, M. A. A. Frota pesqueira, esforço de pesca e a produção pesqueira no Rio Grande do Sul. In: Anais do XIV Congresso da Sociedade Brasileira de Economia, Administração e Sociologia Rural. Londrina, 22-25 de julho de 2007.

Thé, A. P. G.; Ruffino, M. Gestão Compartilhada do uso dos recursos pesqueiros: uma nova possibilidade. In: Kalikoski, D.; Dias Neto, J.; Thé, A.P.G.; Ruffino, M.L.; Marrul Filho, S. (Orgs.). Gestão compartilhada do uso sustentável de recursos pesqueiros: refletir para agir. Brasília: Ibama, 2009. p. 39-109.

Vaz, A. C.; Möller Jr., O. O.; Almeida, T. L. Análise quantitativa da descarga dos rios afluentes da Lagoa dos Patos. Atlântica, 28(1), 13-23, 2006.

Vieira, P. F.; Cazella, A.; Cerdan, C.; Carrière, J. P. (Orgs.). Desenvolvimento Territorial Sustentável no Brasil: subsídios para uma política de fomento. Florianópolis: APED, 2010.

Vooren, C. M.; Chiaradia, A. Seasonal Abundance and Behaviour of Coastal Birds in Cassino Beach, Brazil. Ornitologia Neotropical, 1, 09-12, 1990. 
Vooren, C. M.; Klippel, S.; Galina, A. B. Os elasmobrânquios da área costeira da Plataforma Sul. In: Vooren, C. M.; Klippel, S. (Orgs.). Ações para a conservação de tubarões e raias no Sul do Brasil. Porto Alegre: Igaré Editora, 2005a. p. 113-120.
Vooren, C. M.; Klippel, S. Diretrizes para a conservação de espécies ameaçadas de elasmobrânquios. In: Vooren, C. M.; Klippel, S. (Orgs.). Ações para a conservação de tubarões e raias no Sul do Brasil. Porto Alegre: Igaré Editora, 2005b. p. 213-246. 